\title{
From Chemical to Communication Ecology
}

\section{Gerhard Gries}

Published online: 9 April 2014

(C) Springer Science+Business Media New York 2014

There are myriad reasons to study chemical ecology, whether you work primarily on plants, microbes, invertebrates, or vertebrates, or whether you study entire ecosystems. You may simply enjoy the thrill of discovering new semiochemicals, their biosynthetic pathways, or sensory processing and integration. Or, you may study chemical ecology to address societal needs and to provide semiochemical tools for important applications such as the earliest possible detection of invasive organisms or the earth-friendly control of pest insect populations in orchard settings.

In our lab, for a long time, we have devoted a large body of work to the discovery and application of new semiochemicals. We have even built our own tools to elucidate the structure of complicated trace chemicals, some of which present in such low quantities that we could not obtain spectroscopic data. Concurrently, we have marvelled at the work of colleagues who study the mechanisms of olfaction, the role and structure of odor-binding and receptor proteins, or the genes encoding them. In my undergraduate classes, I have lectured about the fascinating world of insect-plant interactions, enjoying the expression of wonder, awe, and curiosity on the faces of my students. All the reasons that prompted me to enter the field of chemical ecology remain, and the sense of wonder gained by working in this field will never leave me. One thing, however, did change.

That change started in 1999 when Stephen Takács (a PhD student of mine) studied the chemical ecology of webbing clothes moth and when he noticed that male moths wing fan on dry animal pelts. Stephen convincingly demonstrated that the wing fanning sound had a signal function during sexual communication. As a result of his observation, our lab entered a phase of soul searching. Of course, we knew about this phenomenon called multi-modal communication, and that already Darwin (1872) in his "Expression of Emotions in Man and Animals" had argued that animals communicate in multiple modalities. As well, we had read the ground-breaking papers by Rowe and Guildford (1996) and Partan and Marler (1999) on multi-modal signalling. But, curiously, only after we had come across an unexpected non-pheromonal (sound) signal in one of our own research projects did the importance and relevance of multi-modal communication fully "sink in". At the time, we had the option to simply shut out the "alien world" of sound communication or to welcome it into our research portfolio. We opted for the latter. Ever since, the "Takács phenomenon" has emerged in multiple projects, with the non-chemical signal or cue being visual, vibratory, infrared, or even electromagnetic. At each crossroad, we opted to include the new modality into our

G. Gries $(\bowtie)$

Simon Fraser University, Burnaby, British Columbia V5A1S6, Canada

e-mail: gries@sfu.ca research program of multi-modal animal communication ecology. This turned out to become an expensive endeavor and made me doubly aware that you want to never stop learning and integrating new technologies. Indeed, I consider myself the oldest student in the lab! In the process you do, however, run the risk of becoming a "jack of all trades but master of none".

The benefits of studying multi-modal communication signals or foraging cues, in any case, are enormous. To put it in the words of Thomas Baker (1993), you are truly "Learning the Language of Insects [or animals] - and How to Talk Back". This is particularly relevant if your research is funded, at least in part, by industrial sponsors that want solutions to challenging pest problems, irrespective of the type of communication system employed by the target species. If that species happens to be a multi-modal signaller, as many insects, spiders, birds, frogs, lizards, and primates (including ourselves) are, and you study just the chemical signal in their complex communication system, you are bound to learn what may amount to merely a single word in a long sentence, and you may miss the full meaning of the message. In turn, your "talking back" will not be as effective as it could or should be, and you may not be "understood" at all. For instance, to effectively "talk to" stink bugs, you will need to reproduce their long-range pheromone signals and their short-range, substrate-born vibratory signals. Either signal alone will be insufficient to "engage" them in a conversation.

But even if you never want to become a Dr. Doolittle of the animal world, or prefer basic to applied research, there is plenty of reason to expand your studies in chemical ecology to communication ecology, irrespective of your focal area of interest. Evolutionary (chemical) ecologists, for example, are aware that communication by multi- rather than uni-modal (chemical) signals is fundamental to all aspects of mate choice in many taxa, and that mate choice and sexual selection are the key driving forces in speciation. Studying multi-modal communication signals from a neuroscience perspective appears equally appealing. The International Society of Chemical Ecology has outstanding members who have greatly advanced our knowledge of how chemical signals are received, neurally processed, and integrated. Much of their work has been published in the Journal of Chemical Ecology. While all of this is absolutely exciting, imagine the "fireworks display" of an insect brain processing simultaneously (?) the olfactory, visual, and acoustic signals from a prospective mate! I think there is a lot to discover here!

My vision is to continue to move our research from chemical ecology to communication ecology, inviting physicist or biologist experts on vision, audition, and even electromagnetism to contribute to our inter- and multidisciplinary endeavor aimed at understanding multi-modal communication and becoming increasingly better at "talking back". Our first and foremost love affair will always be chemical ecology but we are also more than "flirting" with communication ecology. 